\title{
Hybrid patch propagation and diffusion Technique for Image Inpainting
}

\author{
Vineet Kumar, A. K. Sinha, A. K. Solanki
}

\begin{abstract}
This paper deals with anovel hybrid technique based onpatch propagation and diffusion method for image inpainting. The presented technique is used to reconstruct a damaged image. Image inpainting is a method to fill the hole with the best plausible, which is created due to damage. The novel hybridization technique of diffusion-based and exemplar-based is presented to overcome the existing problem of inpainting. The method is tested on the image dataset of TUM-IID. The performance of present method is measured using quality factor $(Q F)$ analysis, peak signal-noise ratio (PSNR), and comparing similarity by structure similarity index (SSIM). Result demonstrates that the proposed calculation performed better contrast with the existing exemplar-based technique.
\end{abstract}

Index Terms: Exemplar Inpainting, Patch Propagation, patch diffusion, PSNR, QF, SSIM

\section{INTRODUCTION}

The inpainting techniques refer to fill the hole created due to damaging of the picture. It is used to reconstruct the image with the best plausible fill in a gap that is created due to the removal of any unwanted object from the image. It has attained an incredible research scope in the domain of image processing. Presently the area of computer vision has found great scope for image analysis. In this regards, Image inpainting has gained lot of scope to solve many image related issues. Image inpainting is also referred to as image completion process, which recreate the missing parts of an image by reconstructing the existing damaged image to reproduce the original image.An image has many complex features like different texture, different shape of objects that contains many edges, the unusual combination of color, etc.That's why image inpainting techniques are modeled to reconstruct the image with maintaining texture and structure as well.

To solve the problem that exists in Image completion, Techniques are divided into two categories.

a. Texture-based inpainting: In this, missing regions in an image are filled by regenerating the patches from the surroundings.

Revised Manuscript Received on October 5, 2019.

Vineet Kumar, Computer Science \& Engineering, Noida Institute of Engineering \& Technology, Greater Noida, India.

Dr. A. K. Sinha, Director, UST Software India Pvt Ltd., New Delhi, India

Dr. A. K. Solanki, Department of Computer Science \& Engineering, BIET, Jhansi, India. b. Structural based inpainting: It uses the geometric information to fill the missing parts of the image.

Both techniques have limitations to the reconstruction of images. Texture based methods fail to complete the complex structure while structural based methods are not able to fill the large missing region of texture.

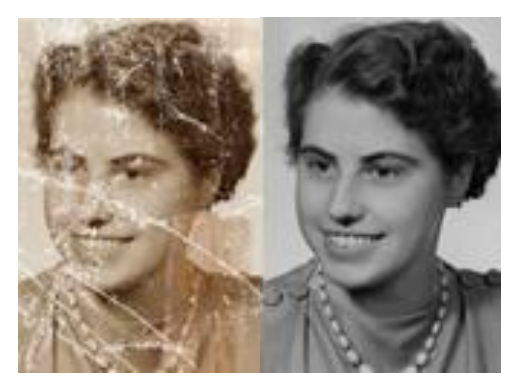

Fig.1a) Damaged Image

b) Inpainted Image

In 2000, Bartalmio et al. [1] proposed the first inpainting technique which used an anisotropic model that propagates the information from surrounding neighborhoods into the epicenter of the missing part through the isophote lines. This method is found suitable for filling the small missing area, but it produces a blurring image due to anisotropic diffusion [8]. In [2], authors have proposed a variational model using second-order partial differential equations which improve the results of previous model. Many researchers have contributed in the same direction $[5,6,7]$. Such methods are also known as diffusion-based methods.

Efors and Leung [11] developed a technique based on copying a pixel from the surroundings of missing part. This technique is based on recreation of missing part by selecting a pixel from the boundary region of the occluded area and finding a similar pixel around it to fill. This method was copying the pixel by pixel to reconstruct the texture. The techniques present in $[17,18,19]$ performed the texture synthesis, which replicates the patch (block) instead of a pixel in the target region. These techniques are classified as Exemplar based techniques. 
The combination of diffusion-based methods and texture synthesis is proposed by Criminisi et al. [13].This method is based on the hybridization of diffusion and texture synthesis. It eliminates the shortcomings of blurriness created during the diffusion-based inpainting and failure occurred in recreation of linear features alike in texture synthesis.Many authors have contributed to the developing of hybrid inpainting techniques.

The existing methods provide a robust mechanism of image completion but cannot handle curved structure ambiguity and not able to produce a consistent result for the nonexistence of similar patches. These methods were difficult to extract complete salient features of complex structures and not intelligent enough to inpainting a large region in a natural image containing complex textures and objects.

\section{LITERATURE SURVEY}

In computer vision theory, Image inpainting refers to the method of recreating the lost part of an image. It is the process that deals to remove objects or scenes from digital pictures and to replace them with the visually likely environment [1]. The various algorithm explored by researchers to reconstruct the missing blocks of an image, and image restoration. Guillemot and Le Meur [28] classified inpainting methods into diffusion and exemplar-based methods

The first algorithm for image inpainting wasproposed by Bertalmio et al. [1] using a diffusion-based approach. This algorithm was based on filling the missing portion by replicating the information from nearby region along the isotropic direction. Subsequently, remarkable research has been done in the course of Image inpainting. In [2], Bertalmio proposed that the variational problem is solved by computing the interpolation, which leads the second-order partial differential equation (PDE) for grey-level and gradient orientation. [3] uses Navier-Strokes equations of fluid dynamics to diffuse isophote by obtaining a similar gradient from the edge of the missing region. In 2003 Bertalmio et al. [4] proposed to fill the hole by filling texture and structure together. These above models are not well suited to fill the sizeable missing region and not able to maintain the textual properties of an image.

Chan and Shen [5] proposed variation model to fill the hole in grey level and color images. The authors proposed a curvature-driven diffusion for non-texture images by computing third-order PDE [6,7,8].A fast-marching method was proposed by Telea[9]. In this method the image gradient helps in image smoothness from boundary pixelsto the region to be inpainted. The technique created the fogginess when a vast inpainting region was to be inpainted.

Texture synthesis presents a vital role in computer vision applications. It is challenging to reconstruct the image with the exact texture if the texture present in an image is damaged.Wei and Levoy [10] proposed a model that generates the missing texture by deterministic search procedure.

The second kind of algorithm which addresses the image inpainting is Exemplar based techniques. Exemplar methods attempt to locate the best patch (a small region of the image) from the known source area and reconstruct the lost parts by duplicating content from the current piece of the image.These algorithms followed two steps. In first step, the patch was selected to filled, and in second step the patch was propagated along with inherent texture and structure. Patch selection is based on specifying the highest priority while propagating the patch relate to finding the most suitable patch from which information of pixel is copied.Harison[12] used the synthesizing process over an image by providing a masked image as input. These methods also cannot reconstruct the damaged complex texture and missing large structure in the image

Criminisi et al. [13] proposed that texture and structure are replicated using exemplar-based texture synthesis. The success rate of these algorithms was depended on the filling criteria of the patch in the target region. Criminisi et al. [14] presented the best-first algorithm based on assigning the confidence value concepts. They synthesized the higher confidence value pixel to the inpainting region. Komodakis and Tziritas [15] proposed a scheduling to solve the inpainting problem by computing the distances between the patch present at the boundary of known and unknown region. In [16], patch located in the boundary of inpainted region and neighborhood windows is examined to minimize to compute many distances. The algorithm presented get improvement in structure propagation without increasing the complexity.

Xu et al. [17] introduced an algorithm that computes the patch sparsity and propagates the patch with fill front filling algorithm. The selection of a patch is based on the higher priority assigned to a patch that contains the sparseness of nonzero similarities of the nearby patch.

Shutao and Zhao [23] used wavelet transform to detect feature, curve fitting for completing the structure then synthesize the texture information and propagate to missing regions but difficult to extract complete salient feature of complex structure of the missing region.

Wallace Casaca et. al. [24] revisited the Image decomposition and transport equation to provide a mechanism to define the selection of patch. In this method, the similarity between the pixel blocks and the order of filling the unknown region are taken care. These methods require various parameters to implement but yield disappointing results for a non-textured image with color variation.

Zhidan et al. [25] gave a color-gradient patch sparsity(CGPS) based approach to provide candidate patch and order of filling. In this approach WCGD and CGSS are used to fulfill the purpose. Exemplar based approach only used the color gradient while this method used both the color and gradient information. This approach is quite useful in maintaining structural coherence and texture. The technique was not intelligent enough to inpainting large regions in a natural image containing complex texture and objects.

An intelligent image inpainting algorithm is proposed to provide a high degree of accurate inpainting results and increase the processing speed of the inpainting method. 
The proposed algorithm works in three phases: (i) assigning the priority to the patch which can reconstruct missing target region by maintaining its textural and structural components (ii) Selection of best suitable candidate patch from the source region. (iii) Fit the selected patch to the target region.

Thispaper comprises of 5 sections.Section 1 refers introduction to the image inpainting techniques. Section 2 deals with literature survey. In Section 3, a context-driven hybrid inpainting technique is proposed. In the proposed technique, the exemplar-based method is integrated with a diffusion-based approach to overcome the critical limitation of both the techniques. Section 3 elaborates on the execution approach of a hybrid technique. The results are concluded in section 4. This section also deals with the comparison of outcomes of proposed model with the results of existing exemplar model. The discussion regarding the results is also included in this section. Section 5 of the paper concludes the of the results obtained.

\section{PROPOSED HYBRID PATCH PROPAGATION USING DIFFUSION}

Hybrid image inpainting can simulate both exemplar and diffusion-based image inpainting techniques. The method depends on finding the best patch for the region to be inpainting and the way that candidate patch is propagated in the target region. The purpose of proposed algorithm is to reduce the processing time and sustain the textual and structural components of the missing target area as we can observe from naked eyes. Following steps are carried out.

Step1. Determine the target region to be fill.

Step2. For each patch compute priority of the patch by applying exemplar-based method.

Step3. Choose the maximal priority patch from the source region for the target region.

Step4. Compute the best fit patch.

Step5. Use the diffusion method to construct the structure of unknown or target region.

\section{Step1. Determine the target regionto fill}

Inpainted region is determined by subtracting the mask region from the original image Let $\boldsymbol{I}$ is an original image and Imask is the target region to be inpaint then an image with mask is obtained by I-Imask.

\section{Step2. Exemplar-based inpainting}

Among the available inpainting algorithm, the exemplar-based methodology is observed to be most useful to reconstruct the large missing region and maintain the texture of the image.This methodology involves two modules. The first module is used to assign the priority to all the nearby patch of pixel present at the boundary of the hole, and the second module is used to select the most suitable matching patch. In this approach, unknown region is filled by spatial information which is computed using the priority and data information of the best selected patch of the nearby region

Let $\mathrm{I}$ is a digitalimage, $\Omega$ is a target region for filling the gap and $\Phi$ is the source region. The objective of the approach is to fill the missing region (target) using boundary information, of the source $\delta \Omega$. Let $\mathrm{p}$ is the center of patch selected at the boundary of source region.

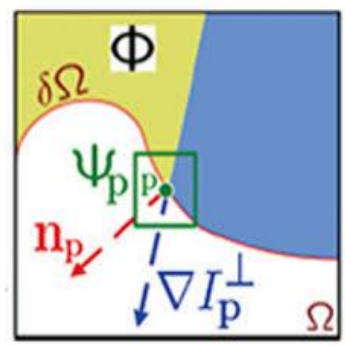

Fig.2: Notation of Exemplar based Method

Step 3. Computing patch priorities:

The patch priority denoted as $\mathrm{P}(p)$ is computed for each patch

$$
P(p)=C(p) \cdot D(p)(1)
$$

Where $\mathrm{C}(\mathrm{p})$ is confidence term is defined as

$$
\mathrm{C}(\mathrm{p})=\Sigma \mathrm{q} \in \Psi_{\mathrm{p}} \cap \Omega^{\prime} \mathrm{C}(\mathrm{q}) /\left|\Psi_{\mathrm{p}}\right| \text { (2) }
$$

and data term $\mathrm{D}(\mathrm{p})$ is defined as

$$
\mathrm{D}(\mathrm{p})=\| \nabla \mathrm{p} \perp \cdot \mathrm{np} \mid \alpha
$$

Where $\Omega$ ' denotes the complement of target region $\Omega$, and $|\Psi \mathrm{p}|$ is patch area, $\mathrm{n}_{\mathrm{p}}$ is the unit vector diagonal to the $\delta \Omega$ at the point $p, \nabla \mathrm{p} \perp$ is an isophote vector and $\alpha(\alpha=255)$ is normalization parameter.

Data Term $\mathrm{D}(\mathrm{p})$ first encourages to synthesize linearstructures and thus safely propagates into the target domain, Confidence term $\mathrm{C}(\mathrm{p})$ represents the set of reliable information surrounding pixel $\mathrm{p}$. Initial value of confidence term is set as $\mathrm{C}(\mathrm{p})=0, \forall \mathrm{p}$ belongs to $\Omega$ and $\mathrm{C}(\mathrm{p})=1, \forall \mathrm{p}$ belongs toI - $\Omega$. The iteration of exemplar-based inpainting is halted if no block is inpainting.

\section{Step 4. Computation of best fit patch}

When the priority of the patch is computed, then it is assigned to each patch. The best-fit patch algorithm is used to select the best-fit matched patch. The best-fit matched patch algorithm is based on the quicksort algorithm. Algorithm sort the patches based on the data obtained in step3.

\section{Step 5:Diffusion-based inpainting:}

After getting best matched patch and determining the region to be fill, a non-linear diffusion method is applied to fill the missing part so that blurring and localization problem is minimized. A process of inhomogeneous diffusion is applied to reduce the spreading of the pixel of the patch and find the more significant plausibility of the existing structural component. 


\section{RESULT AND DISCUSSION}

The proposed hybrid inpainting algorithm is tested on the TUM-IID image data set. The data set contains 17 images of complex structure and texture. Out of 17 images, eight images are taken to test the model.

Three different types of mask images are used to test the efficiency of the algorithm.

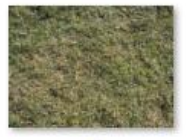

1

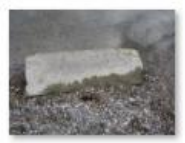

5
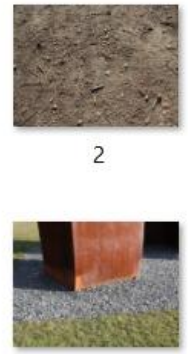
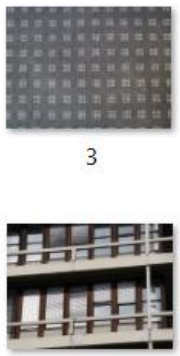

7
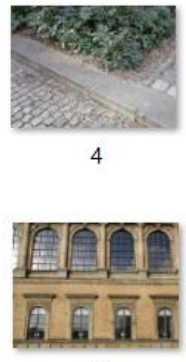

8
Fig. 3. Testing Images of TUM-IID image Dataset

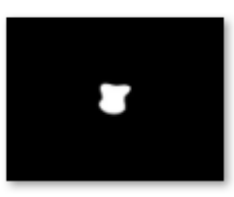

mask1

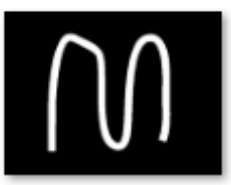

mask2

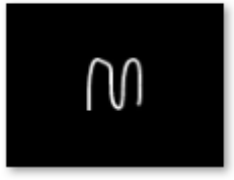

mask3
Fig.4.Different type of Mask Images
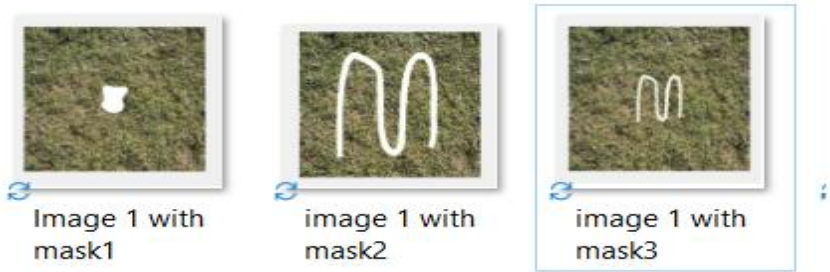

Fig 5.Applying the mask on Image 1

Experiments are carried out on MATLAB 2017a. For the patch selection a quick sort algorithm is designed for which C Mex compiler is used. Proposed hybrid inpainting algorithm performance is evaluated on the three different parameter which are popular in computer vision. The parameters, PSNR to measuring the correctness of images after removal the noise, Quality factor (QF) for measuring correlation of pixel of different images and for finding similarity in structure, structure similarity index (SSIM) are used.

Chosen Parameter PSNR is a ratio of a maximum intensity of the pixel and the corrupted noise that alters the accuracy of its image.

Let image $\mathrm{I}$ is an input image which is passed in to proposed model with masked image $\mathrm{I}_{\text {mask }}$ and we get InP an inpainted image. The size of each image is $\mathrm{Mx} \mathrm{N}$

$$
\begin{gathered}
M S E=\left(\frac{1}{M N}\right) \sum_{i=1}^{M} \sum_{j=1}^{N}(\operatorname{In} P(i, j)-I(i, j))^{2}(4) \\
P S N R=10 \times \log _{10}\left(\frac{255^{2}}{M S E}\right) d B
\end{gathered}
$$

Structural Similarityof the original input image and inpainted Image is verified using SSIM. SSIM is calculated on statistical feature of the original and inpainted image as mean $(\mu)$, variance $\left(\sigma^{2}\right)$ and covariance $(c o v)$

$$
\operatorname{SSTM}\left(x_{z} y\right)=\frac{\left(2 \mu_{1} \mu_{2}+c_{1}\right)\left(2 \operatorname{Cov}_{12}+c_{2}\right)}{\left(u_{1}^{2}+u_{z}^{2}+c_{1}\right)\left(\sigma_{1}^{2}+\sigma_{2}^{2}+c_{2}\right)}
$$

Where $\mathrm{c} 1$ and $\mathrm{c} 2$ are the constant used to keep away from vulnerability. The values of constants depend on image size. The pixel-wise correlation between the images are measured by the quality factor $(\mathrm{QF})$

$$
Q F=C C * \frac{\left(2 \mu_{1} \mu_{2}\right)\left(2 \sigma_{1}+\sigma_{2}\right)}{\left(u_{1}^{2}+u_{2}^{2}\right)\left(\left(\sigma_{1}^{2}+\sigma_{2}^{2}\right)\right.}(7)
$$

Where CC denotesthe correlation coefficient.

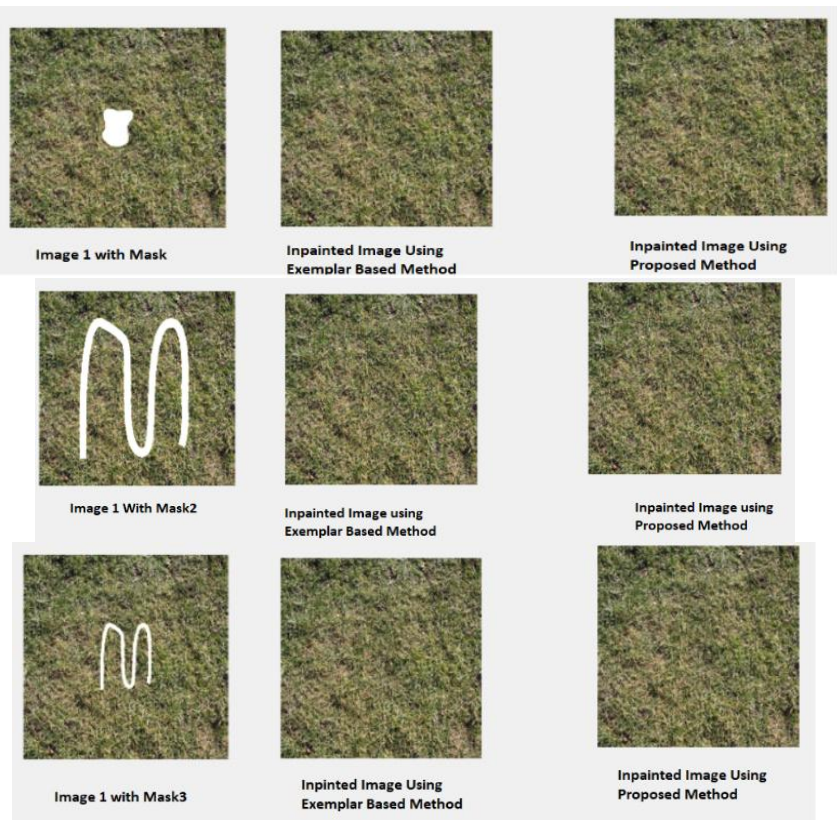

Fig 6. Experimental results on image 1 with different mask

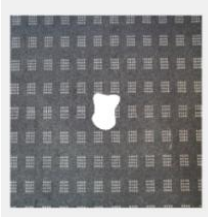

Image 3 With Mask1

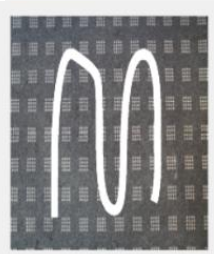

Image 3 with mask2

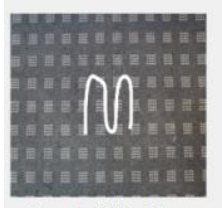

Image 3 with Mask3
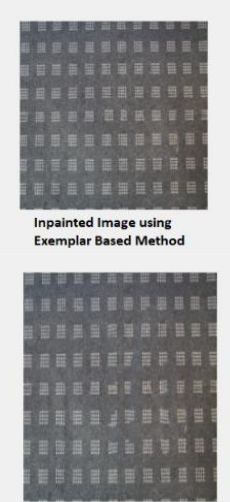

Inpainted Image Using
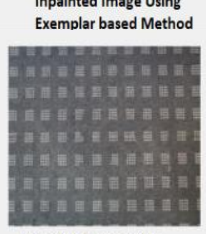

Inpainted Image using
Exemplar Based Method

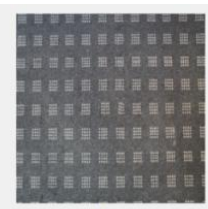

Inpainted Image using
Proposed Method

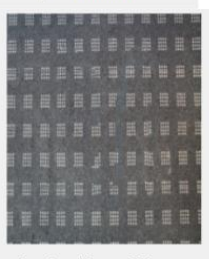

Inpainted Image Using

Proposed Method

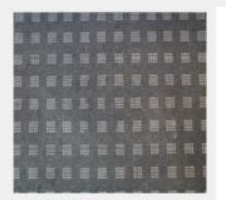

Fig 7. Experimental results on image 3 with different mask

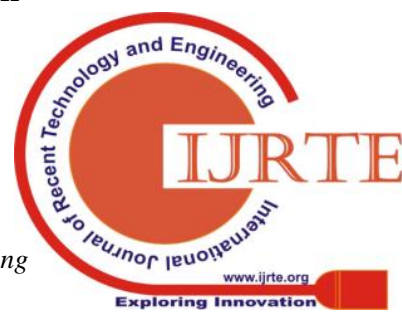


Table 1. Comparative performance analysis of existing exemplar and proposed model on mask1

\begin{tabular}{|c|c|c|c|c|c|c|c|c|c|}
\hline \multirow{2}{*}{ Image } & \multicolumn{3}{|c|}{ PSNR } & \multicolumn{3}{c|}{ SSIM } & \multicolumn{3}{c|}{ QF } \\
\cline { 2 - 10 } & $\begin{array}{c}\text { Exemplar } \\
\text { Based }\end{array}$ & $\begin{array}{c}\text { Proposed } \\
\text { Method }\end{array}$ & $\begin{array}{c}\text { \% } \\
\text { Improvement }\end{array}$ & $\begin{array}{c}\text { Exemplar } \\
\text { Based }\end{array}$ & $\begin{array}{c}\text { Proposed } \\
\text { Method }\end{array}$ & $\begin{array}{c}\text { \% } \\
\text { Improvement }\end{array}$ & $\begin{array}{c}\text { Exemplar } \\
\text { Based }\end{array}$ & $\begin{array}{c}\text { Proposed } \\
\text { Method }\end{array}$ & $\begin{array}{c}\text { \% } \\
\text { Improvement }\end{array}$ \\
\hline $\mathbf{1}$ & 23.703 & 25.865 & 8.358786004 & 0.9923 & 0.9952 & 0.291399 & 0.843 & 0.8982 & 6.14562458 \\
\hline $\mathbf{2}$ & 22.844 & 25.362 & 9.928239098 & 0.9931 & 0.996 & 0.291165 & 0.8456 & 0.9054 & 6.60481555 \\
\hline $\mathbf{3}$ & 16.165 & 24.267 & 33.38690403 & 0.9947 & 0.9991 & 0.440396 & 0.7871 & 0.9596 & 17.9762401 \\
\hline $\mathbf{4}$ & 23.13 & 24.738 & 6.500121271 & 0.994 & 0.9958 & 0.180759 & 0.9349 & 0.9543 & 2.0329037 \\
\hline $\mathbf{5}$ & 23.06 & 23.315 & 1.093716492 & 0.9957 & 0.9963 & 0.060223 & 0.9159 & 0.9148 & -0.12024486 \\
\hline $\mathbf{6}$ & 20.725 & 26.525 & 21.866164 & 0.9905 & 0.9976 & 0.711708 & 0.9133 & 0.9766 & 6.4816711 \\
\hline $\mathbf{7}$ & 23.746 & 29.292 & 18.9334972 & 0.9845 & 0.9957 & 1.124837 & 0.9294 & 0.9801 & 5.17294154 \\
\hline $\mathbf{8}$ & 22.635 & 25.38 & 10.81560284 & 0.9935 & 0.9966 & 0.311058 & 0.9226 & 0.9582 & 3.71529952 \\
\hline
\end{tabular}

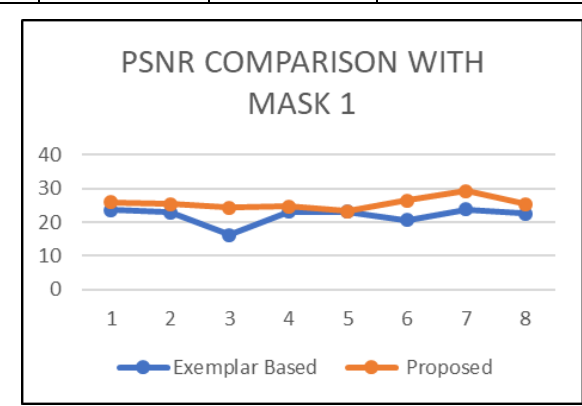

(a)

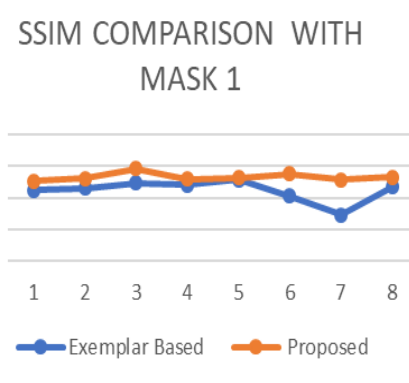

(b)

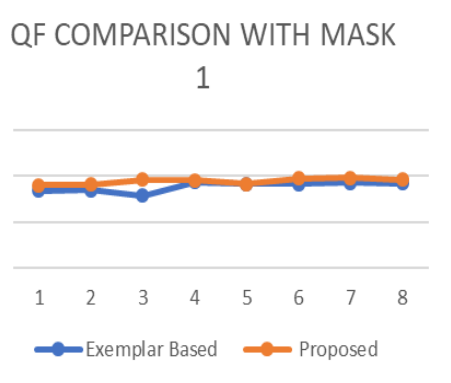

(c)

Fig 8. (a) Comparison graph of PSNRfor exemplar and proposed model, (b) Comparison graph of SSIMfor exemplar and proposed model (c) Comparison graph of QFfor exemplar and proposed model on Mask1.

Table 2. Comparative performance analysis of existing exemplar and proposed model on mask 2

\begin{tabular}{|c|c|c|c|c|c|c|c|c|c|}
\hline \multirow{2}{*}{ Image } & \multicolumn{3}{|c|}{ PSNR } & \multicolumn{3}{c|}{ SSIM } & \multicolumn{3}{c|}{ QF } \\
\cline { 2 - 10 } & $\begin{array}{c}\text { Exemplar } \\
\text { Based }\end{array}$ & $\begin{array}{c}\text { Proposed } \\
\text { Method }\end{array}$ & $\begin{array}{c}\text { \% } \\
\text { Improvement }\end{array}$ & $\begin{array}{c}\text { Exemplar } \\
\text { Based }\end{array}$ & $\begin{array}{c}\text { Proposed } \\
\text { Method }\end{array}$ & $\begin{array}{c}\text { \% } \\
\text { Improvement }\end{array}$ & $\begin{array}{c}\text { Exemplar } \\
\text { Based }\end{array}$ & $\begin{array}{c}\text { Proposed } \\
\text { Method }\end{array}$ & $\begin{array}{c}\text { \% } \\
\text { Improvement }\end{array}$ \\
\hline $\mathbf{1}$ & 16.018 & 17.247 & 7.125876964 & 0.9989 & 0.9991 & 0.020018 & 0.9772 & 0.9826 & 0.54956239 \\
\hline $\mathbf{2}$ & 15.816 & 16.309 & 3.022870808 & 0.9991 & 0.9992 & 0.010008 & 0.9794 & 0.9816 & 0.22412388 \\
\hline $\mathbf{3}$ & 10.482 & 15.708 & 33.2696715 & 0.9992 & 0.9997 & 0.050015 & 0.9648 & 0.9891 & 2.45677889 \\
\hline $\mathbf{4}$ & 16.351 & 14.462 & -13.0618172 & 0.9994 & 0.9991 & -0.03003 & 0.9938 & 0.9906 & -0.32303654 \\
\hline $\mathbf{5}$ & 21.486 & 14.462 & -48.5686627 & 0.9994 & 0.9975 & -0.19048 & 0.9874 & 0.9438 & -4.6196228 \\
\hline $\mathbf{6}$ & 14.322 & 15.966 & 10.29688087 & 0.9992 & 0.9994 & 0.020012 & 0.9922 & 0.9438 & -5.12820513 \\
\hline $\mathbf{7}$ & 18.342 & 24.241 & 24.33480467 & 0.9947 & 0.9987 & 0.400521 & 0.9775 & 0.9942 & 1.67974251 \\
\hline $\mathbf{8}$ & 12.587 & 15.838 & 20.52658164 & 0.9994 & 0.9996 & 0.020008 & 0.9911 & 0.9958 & 0.47198233 \\
\hline
\end{tabular}

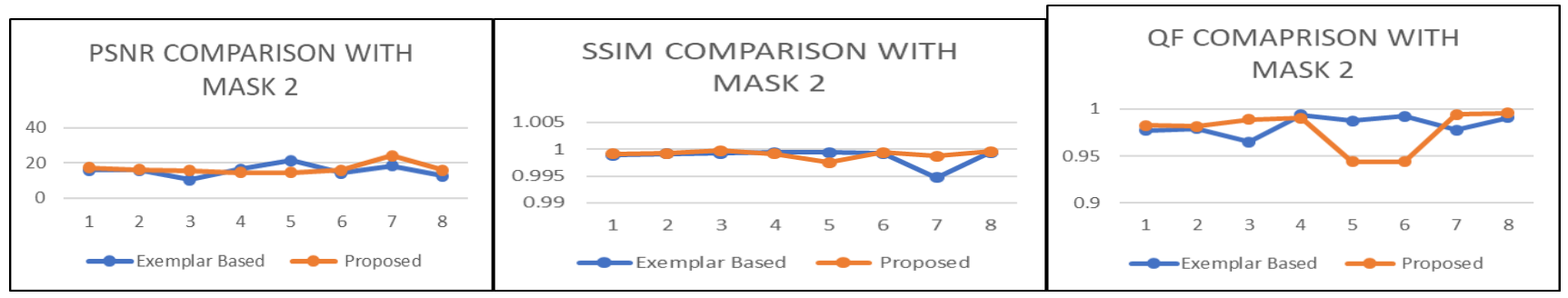

Fig 9. (a) Comparison graph of PSNRfor exemplar and proposed model, (b) Comparison graph of SSIMfor exemplar and proposed model (c) Comparison graph of QFfor exemplar and proposed model on Mask2. 
Hybrid patch propagation and diffusion Technique for Image Inpainting

Table 3. Comparative performance analysis of existing exemplar and proposed model on mask 2

\begin{tabular}{|c|c|c|c|c|c|c|c|c|c|}
\hline \multirow{2}{*}{ Image } & \multicolumn{3}{|c|}{ PSNR } & \multicolumn{3}{c|}{ SSIM } & \multicolumn{3}{c|}{ QF } \\
\cline { 2 - 10 } & $\begin{array}{c}\text { Exemplar } \\
\text { Based }\end{array}$ & $\begin{array}{c}\text { Proposed } \\
\text { Method }\end{array}$ & $\begin{array}{c}\text { \% } \\
\text { Improvement }\end{array}$ & $\begin{array}{c}\text { Exemplar } \\
\text { Based }\end{array}$ & $\begin{array}{c}\text { Proposed } \\
\text { Method }\end{array}$ & $\begin{array}{c}\text { \% } \\
\text { Improvement }\end{array}$ & $\begin{array}{c}\text { Exemplar } \\
\text { Based }\end{array}$ & $\begin{array}{c}\text { Proposed } \\
\text { Method }\end{array}$ & $\begin{array}{c}\text { \% } \\
\text { Improvement }\end{array}$ \\
\hline $\mathbf{1}$ & 17.544 & 20.557 & 14.65681 & 0.9977 & 0.9988 & 0.110132 & 0.952 & 0.9753 & 2.38900851 \\
\hline $\mathbf{2}$ & 15.176 & 19.588 & 22.52399 & 0.9982 & 0.9993 & 0.110077 & 0.9575 & 0.948 & -1.00210971 \\
\hline $\mathbf{3}$ & 10.866 & 18.191 & 40.26717 & 0.9986 & 0.9997 & 0.110033 & 0.9395 & 0.9881 & 4.91853051 \\
\hline $\mathbf{4}$ & 15.442 & 18.44 & 16.25813 & 0.9986 & 0.9993 & 0.070049 & 0.9848 & 0.9923 & 0.75581981 \\
\hline $\mathbf{5}$ & 9.9833 & 15.715 & 36.4728 & 0.9992 & 0.9998 & 0.060012 & 0.9851 & 0.9959 & 1.08444623 \\
\hline $\mathbf{6}$ & 14.48 & 20.272 & 28.57143 & 0.9979 & 0.9994 & 0.15009 & 0.9793 & 0.9944 & 1.51850362 \\
\hline $\mathbf{7}$ & 9.1286 & 25.096 & 63.62528 & 0.9934 & 0.9998 & 0.640128 & 0.9727 & 0.9993 & 2.6618633 \\
\hline $\mathbf{8}$ & 11.308 & 20.393 & 44.5496 & 0.998 & 0.9997 & 0.170051 & 0.9751 & 0.9969 & 2.18677902 \\
\hline
\end{tabular}

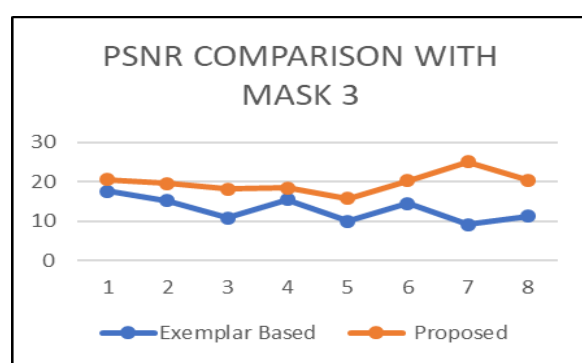

(a)

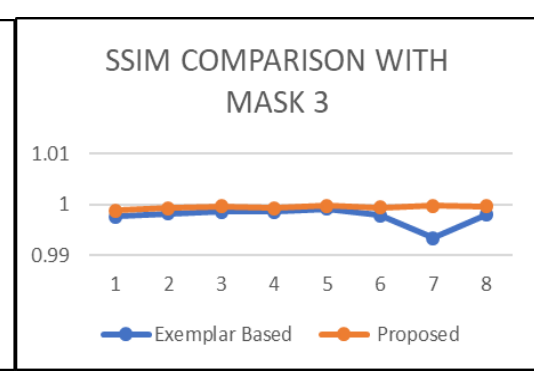

(b)

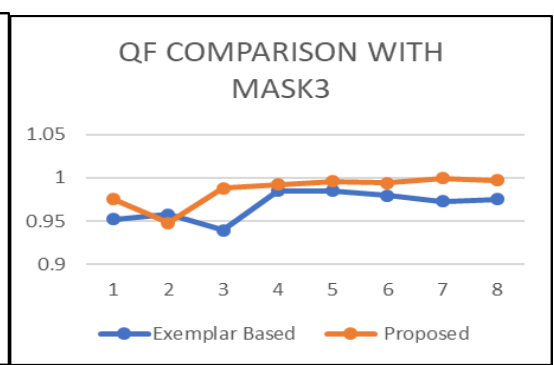

(c)

Fig 10.(a) Comparison graph of PSNRfor exemplar and proposed model, (b) Comparison graph of SSIMfor exemplar and proposed model (c) Comparison graph of QFfor exemplar and proposed model on Mask3.

\section{CONCLUSION}

The hybrid image inpainting technique proposed in this paper offers a viablesolution to identify and remove the undesired objects of an image. It can also be used to reconstruct the lost portion from a digital image at a fast pace without affecting the original image. The proposed technique is based on hybridization of patch propagation and diffusion technique. In this technique the corruptdigital image is mixed with same-sized mask imageto producean uncorrupted image. The proposed algorithm is applied on this uncorrupted image to get plausible original image. The resultsdisplay significant enhancement in the estimation of PSNR, Quality factor, and SSIM over the current techniques. The proposed technique offers good results whenmissing regionsof various sizes are present in the image. It is notable that, when mask size is bigger in complex structured image and it is difficult to reproducetheir texture and structural similarities, the proposed hybrid techniqueworks well to retrieve the texture and structure of missing parts of the object or region. The algorithm offers sharp inpainting capabilities for commercial applications.

\section{REFERENCES}

1. M. Bertalmio, G. Sapiro, V. Caselles, and C. Ballester, "Image inpainting", Proceedings of the ACM SIGGRAPH Conference on Computer Graphics (SIGGRAPH 2000), July 2000, pp. 417-424.

2. C. Ballester, V. Caselles, J. Verdera, M. Bertalmio, and G. Sapiro, "Variational model for filling-in gray level and color images", In Proc. Int. Conf. Computer Vision, pages I: 10-16, Vancouver, Canada, June 2001.
3. M. Bertalmio, A.L. Bertozzi, and G. Sapiro, "Navier-stokes, fluid dynamics, and image and video inpainting", In Proc. Conf. Comp. Vision Pattern Rec., pages I:355-362, Hawai, December 2001.

4. M. Bertalmio, L. Vese, G. Sapiro, and S. Osher, "Simultaneous structure and texture image inpainting", In Proc. Conf. Comp. Vision Pattern Rec., Madison, WI, 2003. http://mountains.ece.umn.edu/ guille/inpainting.htm.

5. T. Chan, J. Shen, "Mathematical models for local non-texture inpaintings", SIAMJ, Appl. Math. 62 (2001) 1019-1043.

6. T. Chan, J. Shen, "Non-texture inpainting by curvature-driven diffusions", J. Vis. Commune. Image Represent. 12 (2001) 436-449.

7. X. Bresson, T. F. Chan, "Fast dual minimization of the vectorial total variation norm and applications to color image processing", Inverse Prob l. Imaging2(2008)455-484.

8. T. Chan and J. Shen, "Local inpainting models and TV inpainting," SIAM J. Appl. Math., vol. 62, no. 3, pp. 1019-1043, 2001.

9. Telea, "An image inpainting technique based on the fast marching method", Journal of Graphics Tools 9(1) (2004) 23-34.

10. Wei, L.-Y., Levoy, M.: 'Fast texture synthesis using tree-structured vector quantization'. ACM SIGGRAPH, July 2000, pp. 479-488.

11. A. Efros, T. K. Leung, "Texture synthesis by non-parametric sampling", in: Proceedings of IEEE International Conference on Computer Vision, 1999, pp.1033-1038.

12. P. Harrison, "A non-hierarchical procedure for resynthesis of complex texture, in: Proceedings of the International conference on Central Europe Computer Graphics, Visualization, and Computer Vision, 2001.

13. Criminisi, P. Perez, K. Toyama, "Object removal by exemplar - based inpainting", in: Proceedings of IEEE Computer Society Conference on Computer Vision and Pattern Recognition, 2003, pp. 721-728.

14. Criminisi, P. Perez, and K. Toyama, "Region filling and object removal by exemplar-based image inpainting," IEEE Trans. Image Process., vol. 13, no. 9, pp. $1200-1212$, Sept. 2004.

15. N. Komodakis and G. Tziritas, "Image completion using global optimization," in IEEE Conference on Computer Vision and Pattern Recognition (CVPR), 2006.

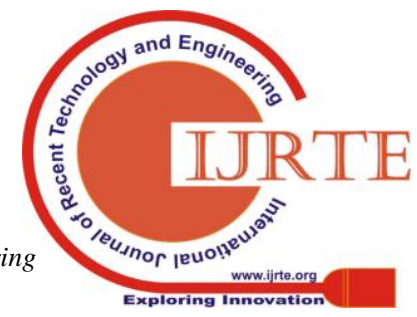


16. N. Kumar, L. Zhang, and S. K. Nayar, "What is a good nearest neighbors algorithm for finding similar patches in images?" in Proc. European Conf. Computer Vision (ECCV), Oct. 2008, pp. 364-378.

17. Barnes, E. Shechtman, A. Finkelstein, and D. B. Goldman, "Patch match: A randomized correspondence algorithm for structural image editing," ACM Trans. Graph., vol. 28, no. 3, pp. 24:1-24:11, July 2009.

18. Z. Xu and J. Sun, "Image inpainting by patch propagation using patch sparsity," IEEE Trans. Image Process., vol. 19, no. 5, pp. 1153-1165, May 2010.

19. Bugeau, M. Bertalmio, V. Caselles, and G. Sapiro, "A comprehensive framework for image inpainting," IEEE Trans. Image Processing, vol. 19, no. 10, pp. 2634-2645, Oct. 2010.

20. O. Le Meur and C. Guillemot, "Super-resolution-based inpainting," in Proc. European Conf. Computer Vision (ECCV), Oct. 2012, pp. 554-567.

21. Barnes, E. Shechtman, D. B. Goldman, and A. Finkelstein, "The generalized Patch Match correspondence algorithm," in Proc. European Conf. Computer Vision (ECCV), Sept. 2010, vol. 6313, pp. 29-43.

22. Wong and J. Orchard, "A nonlocal-means approach to exemplar-based inpainting," in Proc. IEEE Int. Conf. Image Processing (ICIP), 2006, pp. 2600-2603.

23. Shutao Li and Ming Zhao," Image inpainting with salient structure completion and texture propagation", in Proc. of Elsevier Pattern Recognition Letters 32 (2011) 1256-1266

24. Wallace, B. Maurilio,P. D. A. Marcos,G.N. Luis, " Combining anisotropic diffusion, transport equation and texture synthesis for inpainting textured images", in Proc. of Elsevier Pattern Recognition Letters 36 (2014) 36-45

25. Zhidan Li, Hongjie He, Zhongke Yin, Fan Chen, “ A color- gradient patch sparsity based image inpainting algorithm with structure coherence and neighborhood consistency", in Proc. of Elsevier Signal Processing 99 (2014) 116-128.

26. Jiansheng Liu, Hui Liu, ShangpingQiao, Guangxue Yue3, "An Automatic Image Inpainting Algorithm Based on FCM", Hindawi Publishing Corporation the Scientific World Journal Volume 2014 Article ID 201704, 10 pages.

27. RalucaVreja, Remus Brad, "Image Inpainting Methods Evaluation and improvement”, Hindawi Publishing Corporation The Scientific World Journal Volume 2014, Article ID 937845, 11 pages

28. Guillemot, C., Le Meur, O.: 'Image inpainting: overview and recent advances', IEEE Signal Process. Mag., 2014, 31, (1), pp. 127-144

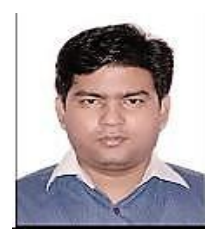

\section{AUTHORS PROFILE}

Vineet Kumar is an associate professor in the department of Computer Science \& engineering in NIET Greater Noida. He has done B.Tech In CSE from GBPUAT Pantnager and MTech in CSE from AKTU Lucknow. He is Pursuing his $\mathrm{PhD}$ in CSE from AKTU Lucknow. He has published 4 research paper in International Journal and 6 research paper in national and international conference. He is life time

member of ISTE.

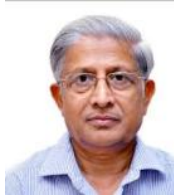

Dr. A. K Sinha is working as a Director UST Software India Pvt. Ltd. New Delhi. He has 37 Year of Teaching Experience in various engineering Colleges of INDIA Dr. Sinha has done BSC (Engg) in Electrical in 1966, MTech in (Control System and Instrumentation in 1976 and PhdIn 1981. He Served as Special Officer in the Department of Science and Technology, Govt. of Bihar Patna and was Director of Maulana Azad College of Engineering \& Technology Patna. Dr. Sinha has supervised 7 Phd thesis and 26 MTech Dissertation.

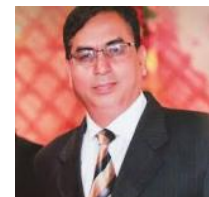

Dr. A. K. Solanki is working as Professor \& Head, Computer Science \& Engineering Department of BIET Jhansi. He has done ME in CSE from NIT Allahabad and $\mathrm{PhD}$ in CSE from Bundelkhand University Jhansi in 2005. Under his supervision 04 Phd Thesis are awarded and $06 \mathrm{PhD}$ research work in progress. he has guided 04 MTech Dissertation and more than 40 . He has published more than 60 research paper in national and international journals/conferences. He is an active member of CSI, ISTE, IEEE, IFEES, ASEE, IACSIT, IAENG. 